\title{
Avaliação de genótipos de morangueiro quanto à qualidade e potencial de armazenamento
}

\author{
Auri Brackmann ${ }^{1}$, Elizandra Pivotto Pavanello ${ }^{2}$, Vanderlei Both ${ }^{3}$, Djeimi Isabel Janisch ${ }^{4}$, \\ Odair José Schmitt ${ }^{5}$, Gustavo Giménez ${ }^{6}$
}

\begin{abstract}
RESUMO
O objetivo deste trabalho foi avaliar a qualidade de cultivares e clones de morangos e o seu potencial de armazenamento sob refrigeração. O delineamento experimental utilizado foi de blocos ao acaso, com quatro repetições de aproximadamente $150 \mathrm{~g}$. Os tratamentos consistiram na avaliação de três cultivares e quatro clones, sendo eles: $\mathrm{LBH}$ 15.1. Arazá, SGH 140.3. Guenoa, LBF 236.3 Yvapitá, LBG 121.4, LBK 16.1, LBK 35.1 e LBK 28.1. As avaliações foram realizadas na colheita e dez dias após o armazenamento a $-0,5^{\circ} \mathrm{C}$, mais dois dias em temperatura de $20^{\circ} \mathrm{C}$. As características avaliadas foram: firmeza de polpa, acidez total titulável, sólidos solúveis totais, relação SST/ATT, ácido ascórbico, podridões e perda de peso. Quanto à qualidade, o cv. Guenoa destaca-se pelo baixo teor de acidez e altos teores de SST e ácido ascórbico, pela alta relação SST/ATT e, também, apresenta melhor conservação, com baixa ocorrência de podridões durante o armazenamento. O cultivar LBF 236.3 Yvapitá e o clone LBK 28.1 apresentam os menores teores de vitamina $\mathrm{C}$ e ratio, o que compromete sua qualidade, bem como menor firmeza de polpa e maior susceptibilidade a podridões, apresentando menor potencial de conservação. Os cultivares e clones que apresentaram melhor qualidade na colheita também apresentaram melhor conservação pós-colheita.
\end{abstract}

Palavras-chave: Cultivares, clones, pós-colheita.

\section{ABSTRACT}

\section{Evaluation of strawberry genotypes on the quality and storage potential}

The objective of this study was to evaluate the quality of strawberry cultivars and clones, and their potential for storage under refrigeration. The experimental design was a randomized block design with four replicates of approximately $150 \mathrm{~g}$ each. The treatments consisted in the evaluation of three cultivars and four clones, namely: LBH 15.1 Arazá, SGH 140.3 Guenoa, LBF 236.3 Yvapitá, LBG 121.4, LBK 16.1, LBK 35.1 and LBK 28.1. Evaluations were made at harvest and after ten days of storage at $-0.5^{\circ} \mathrm{C}$, plus two days at $20^{\circ} \mathrm{C}$. The parameters evaluated were firmness, acidity, soluble solids, TSS/ TTA ratio, ascorbic acid, presence of rotting and weight loss. In terms of quality, cv. Guenoa presented low acidity and high TSS e ascorbic acid, high TSS/TTA ratio and also showed better

\footnotetext{
Recebido para publicação em 24/06/2010 e aprovado em 29/09/2011

${ }^{1}$ Engenheiro-Agrônomo, Doutor. Departamento de Fitotecnia, Universidade Federal de Santa Maria, Centro de Ciências Rurais, Av. Roraima, 1000, Camobi, 97105900. Santa Maria, Rio Grande do Sul, Brasil. auribrackmamm @ gamil.com

${ }^{2}$ Engenheira-Agrônoma, Mestranda. Departamento de Fitotecnia, Universidade Federal de Santa Maria, Centro de Ciências Rurais, Av. Roraima, 1000, Camobi, 97105900 . Santa Maria, Rio Grande do Sul, Brasil. elizandra_pavanello@yahoo.com.br

${ }^{3}$ Engenheiro-Agrônomo, Mestrando. Departamento de Fitotecnia, Universidade Federal de Santa Maria, Centro de Ciências Rurais, Av. Roraima, 1000, 97105900. Santa Maria, Rio Grande do Sul, Brasil. vanderleiboth@yahoo.com.br

${ }^{4}$ Engenheira-Agrônoma, Mestranda. Departamento de Fitotecnia, Universidade Federal de Santa Maria, Centro de Ciências Rurais, Av. Roraima, 1000, Camobi, 97105900 . Santa Maria, Rio Grande do Sul, Brasil. djeimi_agro@yahoo.com.br

${ }^{4}$ Graduando em Agronomia. Departamento de Fitotecnia, Universidade Federal de Santa Maria, Centro de Ciências Rurais, Av. Roraima, 1000, Camobi, 97105900. Santa Maria, Rio Grande do Sul, Brasil. odairschmitt@yahoo.com.br

${ }^{5}$ Engenheiro-Agrônomo, Doutor. Instituto Nacional de Investigación Agropecuaria, Las Brujas, Ruta 48, Km 10, Canelones, Uruguay. gustav_gimenez@yahoo.com.br
}

Rev. Ceres, Viçosa, v. 58, n.5, p. 542-547, set/out, 2011 
conservation, presenting low incidence of decay during storage. The cultivar LBF 236.3 Yvapitá and the clone LBK 28.1 showed the lowest vitamin $\mathrm{C}$ content and TSS/TTA ratio, which compromises its quality. The quick reduction of firmness and increased susceptibility to decay presented by LBF236.3 Yvapitá and LBK 28.1 show a low potential for conservation under refrigeration. Cultivars and clones that had higher quality at harvest, also showed better post-harvest conservation.

Key words: Cultivars, clones, post-harvest.

\section{INTRODUÇÃO}

Da produção total brasileira de morangos, cerca de $50 \%$ é destinada ao consumo in natura (Veiga Júnior, 2005). Por sua cor vermelha brilhante, odor envolvente, textura macia e sabor levemente acidificado, é uma das frutas mais apreciadas no mundo. O sabor característico é proveniente do balanço entre os açúcares, ácidos e substâncias aromáticas especiais. No morango, predominam o ácido cítrico $(0,64$ - 1,15\%) e, dentre os açúcares, a glicose e a frutose $(4,5 \%)$. O morango apresenta altas concentrações de substâncias antioxidantes, como o ácido ascórbico (vitamina C) e o ácido elágico (Atkinson et al., 2006). A vitamina $C$ encontra-se em concentrações próximas a 60 $\mathrm{mg} / 100 \mathrm{~g}$. Estas concentrações são determinadas pelo genótipo, condições de cultivo e forma de armazenamento (Lee \& Kader, 2000).

O morango é uma fruta não climatérica, de alta perecibilidade. Por isso, quando destinado ao consumo in natura, deve ser colhido com sua epiderme apresentando $75 \%$ de coloração vermelho-brilhante (Ronque, 1998). Após a colheita, deve ser refrigerado rapidamente, a fim de minimizar a deterioração. A comercialização do morango é realizada basicamente em bandejas plásticas ou de isopor, cobertas com filme de PVC. Assim, o aspecto visual é o atributo que define a compra (Cantillano, 2004). Frutas com a epiderme brilhante, sem sinais de desidratação ou deterioração são desejáveis. Neste sentido, atendimento às exigências do consumidor e grande resistência ao armazenamento devem ser as principais características a serem levadas em consideração na implantação da cultura.

O sabor e as propriedades nutricionais são características do morango que vêm sendo incrementadas e ganhando importância, tanto nos programas de melhoramento, quanto nos sistemas produtivos. Os cultivares mais utilizados foram desenvolvidos visando a alta produtividade, resistência a doenças, cor e tamanho do fruto, firmeza da polpa e da epiderme, sendo as características organolépticas as menos contempladas no processo de seleção (Camargo \& Passos, 1993; Cardiff, 2000; Vicente et al., 2004). Nos cruzamentos visando ao incremento das parâmetros qualitativos, a fixação de uma característica depende da herdabilidade. Os teores de SST e acidez, os quais determinam em parte o sabor, são controlados por níveis variáveis de variância aditiva e dominância. A firmeza da fruta é uma característica quantitativa com herdabilidade não aditiva. Os teores de vitamina $\mathrm{C}$ apresentam grande variação entre os genótipos, sendo um caráter de alta herdabilidade (Giménez, 2008). As características desejáveis da fruta, visando ao consumo in natura, são a alta firmeza da polpa e da epiderme, a fim de resistirem ao transporte e armazenamento, e o sabor adocicado, com pouco ácido (Camargo \& Passos, 1993). Resende et al. (2008) observaram correlação positiva entre aroma, flavor e teor de sólidos solúveis em morangos. Apontaram, ainda, que morangos com maior relação SST/ATT são preferidos pelo consumidor.

O objetivo deste trabalho foi avaliar o potencial para consumo in natura de cultivares e clones avançados de morangueiro, a partir de características físico-químicas dos frutos e do seu comportamento sob armazenamento refrigerado.

\section{MATERIAIS E MÉTODOS}

O trabalho foi conduzido no Núcleo de Pesquisa em Pós-colheita (NPP), do Departamento de Fitotecnia da Universidade Federal de Santa Maria (UFSM), no ano agrícola de 2008. Cultivares e clones avançados de morangos, oriundos do Programa de Melhoramento da UFSM, em parceria com Instituto Nacional de Investigación Agropecuária INIA, do Uruguai, foram avaliadas quanto à qualidade dos frutos na colheita e após o armazenamento refrigerado, visando à aptidão para consumo in natura. $\mathrm{O}$ cultivo do morangueiro foi realizado na área experimental do Departamento de Fitotecnia. O plantio deu-se no dia primeiro de maio de 2008, em canteiros no solo. Os canteiros foram cobertos com plástico de cor preta e, sob eles, foram construídos túneis baixos, com polietileno de baixa densidade, de 150 micra de espessura. A fertirrigação foi realizada por meio de tubos gotejadores. A dose e a 
frequência do fornecimento de nutrientes foram estabelecidas de acordo com Cocco et al. (2008). Após a colheita, as frutas foram selecionadas, eliminando-se aquelas que se encontravam fora do ponto de maturação ou com ferimentos. O ponto de maturação utilizado foi padrão comercial, de $75 \%$ da epiderme apresentando coloração vermelha.

O delineamento experimental utilizado foi o de blocos ao acaso, com quatro repetições. Os morangos foram acondicionados em bandejas plásticas, envoltas por filme de PVC esticável de $15 \mu \mathrm{m}$, e armazenados em minicâmaras experimentais, com volume de 232 litros. Os tratamentos consistiram na avaliação de três cultivares e quatro clones, sendo eles: LBH 15.1, Arazá, SGH 140.3, Guenoa, LBF 236.3, Yvapitá, LBG 121.4, LBK 16.1, LBK 35.1 e LBK 28.1. Todas as frutas foram armazenadas em atmosfera refrigerada, na temperatura de $-0,5^{\circ} \mathrm{C}$ e com umidade relativa de $96 \%$.

As avaliações foram realizadas no momento da colheita e após dez dias de armazenamento a $-0,5^{\circ} \mathrm{C}$, mais dois dias à temperatura de $20^{\circ} \mathrm{C}$, para simular o período de prateleira dos frutos. As características avaliadas foram: firmeza de polpa, determinada com uso de penetrômetro manual, com uma ponteira de 7,9 mm, sendo o resultado expresso em Newton $(\mathrm{N})$; acidez total titulável, determinada por titulação de $10 \mathrm{~mL}$ de suco em $100 \mathrm{~mL}$ de água destilada, com solução de $\mathrm{NaOH} 0,1 \mathrm{~N}$, até pH 8,1, expressa em percentagem de ácido cítrico; teores de sólidos solúveis totais (SST), determinados por refratometria e apresentados em ${ }^{\circ}$ Brix; ratio (relação SST/ATT), obtido por meio do quociente entre os dois constituintes; ácido ascórbico (vitamina C), determinado por titulometria com iodato de potássio, de acordo com a metodologia proposta pelo Instituto Adolfo Lutz (1985); perda de massa fresca, baseada na diferença de peso dos frutos, entre o início do armazenamento e as datas das avaliações; podridões, por meio de avaliações visuais e expressas em percentual de frutos com sintomas de ataque de patógenos.

Os resultados obtidos foram submetidos à análise de variância. Para a comparação entre médias, adotou-se o teste de Tukey, a 5\% de probabilidade de erro. Os resultados expressos em percentagem foram transformados pela fórmula, arc.sen $\sqrt{\mathrm{x} / 100}$, antes da análise da variância.

\section{RESULTADOS E DISCUSSÃO}

Ao se avaliar a qualidade, no momento da colheita, dos três cultivares e dos quatro clones em estudo, observaram-se variações quanto às qualidades físico-químicas e nutricionais. A acidez titulável, representada pela percentagem de ácido cítrico, foi menor no cultivar SGH 140.3. Guenoa e no clone LBG 121.4, que diferiram estatisticamente dos demais (Tabela 1). O cv. Guenoa e os clones LBG 121.4, LBK 16.1 e LBK 35.1 apresentaram os maiores teores de sólidos solúveis totais (SST) (Tabela 1). A amplitude entre os valores de SST, entre os cultivares, no momento da colheita, foi de $1,73^{\circ} \mathrm{Brix}$, mantendo-se o efeito do genótipo sobre este fator. Os valores de SST foram superiores aos encontrados, por outros autores, nas condições de cultivo do Rio Grande do Sul. Cantillano et al. (2008) observaram valores de 6; 6,8 e $7^{\circ}$ Brix para os cultivares Aromas, Ventana e Camino Real, respectivamente, nas condições de Caxias do Sul. Malgarim et al. (2006), na região de Pelotas, obtiveram frutas $100 \%$ maduras do cv. Camarosa, com $7,35^{\circ}$ Brix.

A relação SST/ATT foi maior para o cv. SGH 140.3. Guenoa, não diferindo do clone LBG 121.4 (Tabela 2). Shaw (1990) afirma que o sabor do morango está condicionado, principalmente, pelo balanço entre os sólidos solúveis e a acidez titulável. Uma alta relação SST/ATT confere às frutas um melhor equilíbrio entre o doce e o ácido, conferindo sabor mais agradável, tornando-as mais atrativas. Resende et al. (2008), avaliando a relação SST/ATT de diferentes cultivares de morango, e relacionando-a com a aceitação por parte do consumidor, observaram que as maiores relações SST/ATT estão associadas à melhor percepção de flavor da fruta. Estes autores encontraram a maior relação para o cv. Camp-Dover, com valor de 13,50. Assim, provavelmente, os cultivares, citados anteriormente, com ratios de 13,67 e 12,83, para Guenoa e clone LBG 121,4, respectivamente, terão boa aceitação pelo consumidor.

Por causa da importância do morango como fonte de vitamina $\mathrm{C}$ na dieta humana, seu conteúdo foi analisado nos cultivares em estudo, sendo que, no momento da colheita, o cv. SGH 140.3. Guenoa foi estatisticamente superior aos demais genótipos (Tabela 2). Os menores valores foram registrados para o cv. LBF 236.3. Yvapitá, não diferindo estatisticamente apenas dos clones LBK 28.1 e LBG 121.4. Em se tratando de firmeza de polpa, no momento da colheita, o clone LBK 28.1 apresentou os maiores valores, não diferindo estatisticamente do cv. LBH 15.1. Arazá (Tabela 3). Levando-se em consideração os parâmetros de qualidade estudados, pode-se dizer que o cv. SGH 140.3. Guenoa foi o que mais se destacou, apresentando maiores valores de SST/ATT e de vitamina $\mathrm{C}$.

Ao avaliar o potencial de conservação dos cultivares e clones, após o armazenamento por 10 dias a $-0,5^{\circ} \mathrm{C}$, mais dois dias a $20^{\circ} \mathrm{C}$, a acidez titulável foi menor no cv. SGH 140.3. Guenoa e no clone LBG 121.4, não diferindo estatisticamente do cv. LBH 15.1. Arazá e do clone LBK 35.1. No entanto, todos os cultivares e clones avaliados apresentaram menores valores de acidez após o armazenamento, como já havia sido comprovado por Holcroft \& Kader (1999), durante o armazenamento de morangos cv. Selva. Esse comportamento é justificado pela utilização dos ácidos orgânicos como substrato, durante o processo respiratório. Na colheita, os teores de SST foram maiores no 
cv. SGH 140.3. Guenoa, não diferindo dos clones LBK 16.1 e LBK 35.1, com valores oscilando entre $7,55^{\circ} \mathrm{Brix}$ e 7,06 ํix (Tabela 1). O teor de sólidos solúveis dá um indicativo da quantidade de açúcares existentes na fruta, embora outros compostos, como os ácidos, as vitaminas, os aminoácidos e algumas pectinas também façam parte, em menores proporções, da composição dos sólidos solúveis da fruta (Kluge et al., 2002).
A relação SST/ATT, seguindo a mesma tendência do momento da colheita, continuou maior, após o armazenamento, para os morangos do cv. 'SGH 140.3. Guenoa' e LBG 121.4, não diferindo do clone LBK 35.1 (Tabela 2). De modo geral, a relação SST/ATT apresentou maiores valores após o armazenamento e isto se deve a uma maior degradação dos ácidos, no processo respiratório das frutas, durante a conservação, uma vez que os SST não

Tabela 1. Teores de ATT e de SST de cultivares e clones de morangos, avaliados na colheita e após armazenamento refrigerado por 10 dias a $-0,5^{\circ} \mathrm{C}$ e mais 2 dias a $20^{\circ} \mathrm{C}$

\begin{tabular}{|c|c|c|c|c|}
\hline \multirow{2}{*}{ Tratamento } & \multicolumn{2}{|c|}{ ATT (\% de ácido. cítrico) } & \multicolumn{2}{|c|}{ SST $\left({ }^{\circ}\right.$ Brix $)$} \\
\hline & Colheita & Após armaz. & Colheita & Após armaz. \\
\hline LBH 15.1 Arazá & $0,79 b^{*}$ & $0,59 \mathrm{bc}$ & $6,62 \mathrm{bc}$ & $5,97 \mathrm{e}$ \\
\hline SGH 140.3 Guenoa & $0,59 \mathrm{c}$ & $0,56 \mathrm{c}$ & 7,93 a & $7,55 \mathrm{a}$ \\
\hline LBF 236.3 Yvapitá & $0,92 \mathrm{a}$ & $0,67 \mathrm{ab}$ & $6,45 \mathrm{bc}$ & $6,50 \mathrm{cde}$ \\
\hline LBG 121.4 & $0,58 \mathrm{c}$ & $0,52 \mathrm{c}$ & $7,30 \mathrm{abc}$ & $6,80 \mathrm{bcd}$ \\
\hline LBK 16.1 & $0,84 \mathrm{ab}$ & $0,69 \mathrm{ab}$ & $7,42 \mathrm{ab}$ & $7,06 \mathrm{abc}$ \\
\hline LBK 35.1 & $0,78 \mathrm{~b}$ & $0,60 \mathrm{abc}$ & 7,93 a & $7,42 \mathrm{ab}$ \\
\hline LBK 28.1 & $0,84 \mathrm{ab}$ & $0,70 \mathrm{a}$ & $6,20 \mathrm{c}$ & $6,25 \mathrm{de}$ \\
\hline Média & 0,76 & 0,62 & 7,12 & 6,79 \\
\hline $\mathrm{CV}(\%)$ & 5,17 & 7,09 & 7,25 & 4,70 \\
\hline
\end{tabular}

*Médias, seguidas pela mesma letra na vertical, não diferem estatisticamente pelo Teste de Tukey a 5\% de probabilidade.

Tabela 2. Relação SST/ATT e vitamina C de cultivares e clones de morangos, avaliados na colheita e após armazenamento refrigerado por 10 dias a $-0,5^{\circ} \mathrm{C}$ e mais 2 dias a $20^{\circ} \mathrm{C}$

\begin{tabular}{|c|c|c|c|c|}
\hline \multirow{2}{*}{ Tratamento } & \multicolumn{2}{|c|}{ Ratio (SST/ATT) } & \multicolumn{2}{|c|}{ Vit. C ( mg vit C/100g) } \\
\hline & Colheita & Após armaz. & Colheita & Após armaz. \\
\hline LBH 15.1 Arazá & $8,40 \mathrm{~cd}^{*}$ & $10,06 \mathrm{c}$ & $60,9 \mathrm{bc} *$ & $58,3 \mathrm{bc}$ \\
\hline SGH 140.3 Guenoa & $13,67 \mathrm{a}$ & 13,44 a & 76,4 a & $75,0 \mathrm{a}$ \\
\hline LBF 236.3 Yvapitá & $7,06 \mathrm{~d}$ & $9,87 \mathrm{c}$ & $50,8 \mathrm{~d}$ & $45,1 \mathrm{~d}$ \\
\hline LBG 121.4 & $12,83 \mathrm{ab}$ & $13,02 \mathrm{a}$ & $53,7 \mathrm{~cd}$ & $49,6 \mathrm{~cd}$ \\
\hline LBK 16.1 & $8,88 \mathrm{~cd}$ & $10,27 \mathrm{bc}$ & $60,0 \mathrm{bc}$ & $58,2 \mathrm{bc}$ \\
\hline LBK 35.1 & $10,19 \mathrm{bc}$ & $10,42 \mathrm{ab}$ & $63,1 \mathrm{~b}$ & $60,7 \mathrm{~b}$ \\
\hline LBK 28.1 & $7,41 \mathrm{~cd}$ & $8,93 \mathrm{c}$ & $52,9 \mathrm{~cd}$ & $47,7 \mathrm{~d}$ \\
\hline Média & 9,78 & 11,14 & 59,69 & 56,38 \\
\hline $\mathrm{CV}(\%)$ & 12,99 & 9,05 & 5,77 & 7,56 \\
\hline
\end{tabular}

*Médias, seguidas pela mesma letra na vertical, não diferem estatisticamente pelo Teste de Tukey a 5\% de probabilidade.

Tabela 3- Firmeza, podridão e perda de peso em diferentes cultivares e clones de morango, armazenados em atmosfera refrigerada por 10 dias a $-0,5^{\circ} \mathrm{C}$ e mais 2 dias a $20^{\circ} \mathrm{C}$

\begin{tabular}{|c|c|c|c|c|}
\hline \multirow{2}{*}{ Tratamento } & \multicolumn{2}{|c|}{ Firmeza de polpa $(\mathbf{N})$} & \multirow{2}{*}{$\begin{array}{l}\text { Podridão (\%) } \\
\text { Após armaz }\end{array}$} & \multirow{2}{*}{$\begin{array}{c}\text { Perda de peso }(\%) \\
\text { Após armaz }\end{array}$} \\
\hline & Colheita & Após armaz. & & \\
\hline LBH 15.1 Arazá & $10,43 a b$ & $9,14 \mathrm{~b}$ & $55,0 \mathrm{ab}$ & $1,21 \mathrm{a}$ \\
\hline SGH 140.3 Guenoa & $9,36 \mathrm{bc}$ & $8,58 \mathrm{bc}$ & $21,4 \mathrm{c}$ & $1,19 \mathrm{a}$ \\
\hline LBF 236.3 Yvapitá & $9,53 \mathrm{bc}$ & $7,48 \mathrm{~cd}$ & $75,1 \mathrm{a}$ & $1,19 \mathrm{a}$ \\
\hline LBG 121.4 & $8,41 \mathrm{c}$ & $6,64 \mathrm{~d}$ & $47,2 \mathrm{~b}$ & $1,20 \mathrm{a}$ \\
\hline LBK 16.1 & $9,29 \mathrm{bc}$ & $8,57 \mathrm{bc}$ & $40,8 \mathrm{bc}$ & $1,24 \mathrm{a}$ \\
\hline LBK 35.1 & $10,25 \mathrm{~b}$ & $9,05 \mathrm{~b}$ & $41,2 \mathrm{bc}$ & $1,25 \mathrm{a}$ \\
\hline LBK 28.1 & $12,10 \mathrm{a}$ & $10,69 \mathrm{a}$ & $63,2 \mathrm{ab}$ & $1,17 \mathrm{a}$ \\
\hline Média & 9,91 & 8,59 & 49,12 & 1,21 \\
\hline $\mathrm{CV}(\%)$ & 7,21 & 7,22 & 14,56 & 5,17 \\
\hline
\end{tabular}

*Médias, seguidas pela mesma letra na vertical, não diferem estatisticamente pelo Teste de Tukey a 5\% de probabilidade. 
apresentaram grandes alterações durante este período, concordando com Brackmann et al. (2001). Esses autores observaram, em morangos do cv. Oso Grande, que os teores de SST, em geral, não sofreram modificações com o tempo de armazenamento, a ponto de prejudicar o sabor. Cantillano et al. (2008) encontraram valores de SST/ ATT entre 8 e 9,5, para os cultivares Camino Real, Aromas e Ventana, cultivados na Serra Gaúcha, submetidos ao armazenamento refrigerado. Os valores obtidos, na maior parte dos clones avaliados neste experimento, são similares ou superiores aos encontrados por esse autor, confirmando seu potencial de aceitação pelo mercado consumidor.

Após o armazenamento, os teores de vitamina $\mathrm{C}$ foram maiores no cv. SGH 140.3. Guenoa (Tabela 2). Também foi possível verificar que houve diminuição dos valores de vitamina $C$, na avaliação após o armazenamento, em relação aos do momento da colheita, tendo sido esta relação menor para o cv. SGH 140.3. Guenoa, com perda de apenas $1,96 \%$ no período. De acordo com Cheftel et al. (1983), a oxidação da vitamina C é acelerada pela ação das enzimas ascorbato oxidase ou peroxidase, durante o armazenamento de frutos e hortaliças. A vitamina $\mathrm{C}$ é a mais instável das vitaminas, por ser sensível aos agentes físico-químicos, como luz, oxigênio e calor, sendo que altas temperaturas e longos períodos de estocagem aceleram sua perda.

O clone LBK 28.1 apresentou a maior firmeza de polpa após o armazenamento (Tabela 3). No entanto, os demais cultivares e clones, mesmo com firmeza de polpa inferior, ainda continuaram com valores aceitáveis para serem comercializados, superiores aos valores de 5,23 a 6,48, encontrados por Cantillano \& Schünemann (2008) para os cultivares Camarosa e Camino Real, em condições de armazenamento semelhantes às deste trabalho. A manutenção da firmeza da polpa dos morangos é um importante atributo de qualidade no manejo pós-colheita. Frutos mais firmes, em geral, estão associados a melhores conservação e aspecto visual, sendo, portanto, preferidos pelos consumidores.

O cultivar SGH 140.3. Guenoa apresentou o menor percentual de podridões, sendo que apenas $21,4 \%$ dos frutos apresentavam alguma lesão provocada por fungos, após o período de 10 dias de armazenamento, a $0,5^{\circ} \mathrm{C}$, mais dois dias, a $20^{\circ} \mathrm{C}$. Este cultivar destacou-se nas características avaliadas no presente trabalho. Em cultivos anteriores, no mesmo local, o cultivar também apresentou características físico-químicas relevantes, o que sugere ser este um material estável. Além disso, apresentou bom potencial produtivo para a região, destacando-se pelo tamanho e formato de fruto, fatores determinantes para sua aceitação pelos consumidores (Giménez, 2008). Os clones LBK 16.1 e LBK 35.1 também apresentaram baixa incidência de podridões, não diferindo estatisticamente da cv. Guenoa (Tabela 3). A maior ocorrência de podridões deu-se nos cultivares LBF 236.3. Yvapitã, LBH 15.1. Arazá e no clone LBK 28.1, apesar de este último clone apresentar maior firmeza de polpa. Durante o período de armazenamento, os genótipos não apresentaram diferenças quanto à perda de massa fresca, sendo que eles perderam entre 1,17 e 1,25\% de peso, até o momento das avaliações (Tabela 3). Esses resultados são satisfatórios, uma vez que, de acordo com García et al. (1998), a máxima perda de peso tolerada, comercialmente, para morangos é de $6 \%$. A perda de água é um acelerador da senescência das frutas, acarretando maior taxa de desintegração da membrana, perda do conteúdo celular e, consequentemente, murchamento e perda da suculência.

\section{CONCLUSÕES}

Dentre os clones estudados, LBG 121.4 e LBK 35.1 destacaram-se pela alta relação SST/ATT, o que os torna mais atrativos, em termos de sabor, apresentando-se promissores para o programa de melhoramento.

O cultivar SGH 140.3. Guenoa foi o que apresentou a melhor qualidade físico-química, além de maior potencial de conservação, graças, principalmente, à baixa susceptibilidade a podridões. Considerando o conjunto das características analisadas neste estudo e dados os antecedentes de alta produtividade, o cultivar SGH 140.3 Guenoa pode ser recomendado para sua utilização comercial no RS.

\section{REFERÊNCIAS}

Atkinson CJ, Dodds PAA, Ford YY, Mière JLE, Taylor JM, Blake PS \& Paul N (2006) Effects of cultivar, fruit number, and reflected photosynteticaly active radiation on Fragaria $x$ ananassa productivity and fruit ellagic acid and ascorbic acid concentrations. Annals of Botany, 97:429-441.

Brackmann A, Hunsche M, Waclawovsky AJ \& Donazzolo J (2001) Armazenamento de morangos cv. Oso Grande (Fragaria ananassa L.) sob elevadas pressões parciais de $\mathrm{CO}_{2}$. Revista Brasileira de Agrociência, 7:10-14.

Camargo LS \& Passos FA (1993) Morango. O melhoramento de plantas no Instituto Agronômico. $1^{\mathrm{a}} \mathrm{Ed}$. Campinas, Instituto Agronômico, 524p.

Cantillano RFF (2004) Fisiologia e manejo da colheita e póscolheita de morangos. In: Simpósio Nacional do Morango, Pelotas. Documento 124, Embrapa Clima Temperado. p.146160 .

Cantillano RFF, Castañeda LMF, Treptow RO \& Schunemann APP (2008) Qualidade físico-química e sensorial de cultivares de morango durante o armazenamento refrigerado. Pelotas, Embrapa Clima Temperado, 29p.

Cantillano RF F \& Schünemann APP (2008) Qualidade pós-colheita de morangos 'Camarosa' e 'Camino Real' em Atmosfera Controlada. In. Congresso Brasileiro de Fruticultura, Vitória. Anais, Sociedade Brasileira de Fruticultura. CD-ROM. 
Cardiff F (2000) Enhancement of flavour in strawberries grown under protection. In:National soft fruit conference, Teagasc. Proceedings, Irish soft fruit grower's association and BORD BIA, p. 2-8

Cheftel JC \& Cheftel H (1983) Introdución a la bioquimica de los alimentos. $2^{\mathrm{a}}$ ed. Zaragosa, 333p.

Cocco C, Oliveira CS, Jänisch DI, Schmitt OJ \& Andriolo JL (2008) Fertirrigação do morangueiro cultivado no solo. Universidade Federal de Santa Maria, 4p.

García JM, Medina RJ \& Olías JM (1998) Quality of strawberries automatically packed in different plastic films. Journal of Food Science, 63:1037-1041.

Giménez GF (2008) Seleção e multiplicação de clones de morangueiro (Fragaria $x$ ananassa Duch.). Tese de Doutorado. Universidade Federal de Santa Maria, 119p.

Holcroft DM \& Kader AA (1999) Carbon dioxide-induced changes in color and anthocyanin synthesis of stored strawberry fruit. HortScience, 34:1244- 1248.

Instituto Adolfo Lutz (1985) Normas analíticas do instituto Adolfo Lutz: métodos químicos e físicos para a análise de alimentos. São Paulo, IAL, 533p.

Kluge RA, Nachtigal JC, Fachinello JC \& Bilhalva AB (2002) Fisiologia e manejo pós-colheita de frutas de clima temperado. $2^{\circ}$ ed. Campinas, Livraria e Editora Rural. 214p.
Lee SK \& Kader AA (2000) Preharvest and postharvest factors influencing vitamin $\mathrm{C}$ content of horticultural crops. Postharvest Biology and Technology, 20:207-220.

Malgarim MB, Tibola CS, Zaicowisk C, Ferri VC \& Silva PR (2006) Modificação da atmosfera e resveratrol na qualidade pós-colheita de morangos cv. Camarosa. Revista Brasileira de Agrociência, 12:67-70.

Resende JTV, Camargo LKP, Argandoña EJS, Marchese A \& Camargol CK (2008) Sensory analysis and chemical characterization of strawberry fruits. Horticultura Brasileira, 26:371-374.

Ronque VER (1998) A cultura do morangueiro. 1a ed. Curitiba, Emater. 206p.

Shaw DV (1990) Response to selection and associated changes in genetic variance for soluble solids and titratable acids contents in strawberries. Journal of American Society Horticultural Science, 115:839-843.

Veiga Junior WG (2005) O morango e sua evolução comercial. In: Carvalho SP (Coord.). Boletim do morango: cultivo convencional, segurança alimentar, cultivo orgânico. Belo Horizonte, Ceasa Minas. 160p.

Vicente E, Gimenez G, Manzzioni A \& Cabot M (2004) Avances del programa de mejoramiento genético de frutilla en Uruguay. In.: Simpósio nacional de morango. Pelotas. Palestras, Embrapa Clima Temperado. p.38-45. 